ROM $2 \mathrm{~F}-99 / 30$

MRI-PHY/P990927

HEP-TH/9909107

\title{
D-branes on Fourfolds with Discrete Torsion
}

\author{
Subir Mukhopadhyay * \\ Mehta Research Institute of Mathematics and Mathematical Physics, \\ Chhatnag Road, Jhusi, Allahabad 211 019, India. \\ $\&$ \\ Koushik Ray ${ }^{\dagger}$ \\ Dipartimento di Fisica, \\ Università di Roma "Tor Vergata", INFN - Sezione di Roma "Tor Vergata", \\ Via della Ricerca Scientifica, 1, 00173 Rome, Italy
}

\begin{abstract}
We study D1-branes on the fourfold $\mathbb{C}^{4} /\left(\mathbb{Z}_{2} \times \mathbb{Z}_{2} \times \mathbb{Z}_{2}\right)$, in the presence of discrete torsion. Discrete torsion is incorporated in the gauge theory of the D1-branes by considering a projective representation of the finite group $\mathbb{Z}_{2} \times \mathbb{Z}_{2} \times \mathbb{Z}_{2}$. The corresponding orbifold is then deformed by perturbing the F-flatness condition of the gauge theory. The moduli space of the resulting gauge theory retains a stable singularity of codimension three.
\end{abstract}

*E-mail: subirm@mri.ernet.in [Address after 24 Sept, 1999 : Fysikum, Stockholm university, Box 6730, S-113 46, Stockholm, Sweden]

${ }^{\dagger}$ E-mail: koushik@roma2.infn.it 


\section{Introduction}

D-branes provide a geometric means to studying orbifold singularities and their desingularisations, as the moduli space of D-branes reproduces the space in which the D-branes are embedded into. Within the scope of string theory, a generalisation of orbifold singularities, when possible, is to turn on a discrete torsion [1], 2]. String theory on an orbifold of the $n$-dimensional complex space $\mathbb{C}^{n}$, e.g. $\mathbb{C}^{n} / G$, where $G$ is a finite group, admits discrete torsion if the second cohomology group of $G$, viz. $H^{2}(G, U(1))$, is non-trivial. At this point, let us recall that here we are considering D-branes on non-compact spaces, which may serve as local models of a compact target space of string theory near a singularity. In the conformal field theoretic description of the string world-sheet, turning on a discrete torsion is tantamount to assigning a non-vanishing weight or phase to the contribution to the string partition function arising in the twisted sector.

In studying a closed string theory on an orbifold in absence of discrete torsion, one can estimate the contributions to the partition function arising in the untwisted and the twisted sectors of the theory, by implementing the quotient in the path integral of the theory. The resulting spectrum, which is the sum of all these contributions, turns out to coincide with that of the theory on the corresponding smooth manifold [3].

The presence of discrete torsion alters the contribution from the twisted sector of the theory. The resulting theory is still consistent as a string theory, but no more a string theory on a blown up manifold. Indeed, the resulting theory might be a consistent string theory on a singular target space [2]. Moreover, the modes in the twisted sector correspond to (partial) deformation of the complex structure of the orbifold, not to the blowing up of the Kähler class. Now that the target space of string theory can be simulated as the moduli space of D-branes, a natural question is whether one can incorporate discrete torsion in this picture. This has been answered in the affirmative in some examples [4, 5]. In this article we will find one more example of this kind.

In terms of the supersymmetric gauge theories used to describe the theory on the world-volume of D-branes, discrete torsion is incorporated in the action of the quotienting group on the position as well as the gauge degrees of freedom carried by the brane. In the presence of discrete torsion one is led to choose a projective representation of the group [ 4 , 5], to be contrasted with the linear representation used when discrete torsion is absent. The resolution of the orbifold in the absence of discrete torsion is effected by adding a Fayet-Iliopoulos term in the gauge theory, thereby perturbing the D-term of the gauge theory. In the presence of discrete torsion, the moduli for the deformation of the singularity are purveyed by parameters appearing in the perturbation of the F-term of the theory. In either case, the choice of the perturbations of the F- and D-terms are guided by the twisted sector of closed string theory in the presence and absence of discrete torsion, respectively [4] 8 ].

D-branes on the three-dimensional orbifold $\mathbb{C}^{3} /\left(\mathbb{Z}_{2} \times \mathbb{Z}_{2}\right)$ have been studied in the absence of discrete torsion [9-11], and in its presence as well [4,5,12]. In the absence of discrete torsion, the moduli space of a D-brane on $\mathbb{C}^{3} /\left(\mathbb{Z}_{2} \times \mathbb{Z}_{2}\right)$ is a blown down conifold. Adding Fayet-Iliopoulos terms in 
the gauge theory corresponds to partial [11] or complete [9, 10] resolution of the singularity, depending on the non-vanishing combinations of the Fayet-Iliopoulos parameters. However, the scenario is rather different in the presence of discrete torsion [4, 5]. In this case, the twisted sector of string theory provides modes not to resolve the singularity, but to deform it. Yet, the moduli space turns out to contain a stable double-point singularity or node, while the codimension-two singularities are deformed away by these modes. This signals a deficiency of certain modes in the twisted sector of the closed string theory corroborating with earlier findings [2]].

In the present article we will concern ourselves with analysing a D1-brane or D-string on $\mathbb{C}^{4} /\left(\mathbb{Z}_{2} \times\right.$ $\left.\mathbb{Z}_{2} \times \mathbb{Z}_{2}\right)$ in the presence of discrete torsion. D1-branes on this space without discrete torsion has been studied earlier [13]. In the absence of discrete torsion, the analysis of the moduli space of D1-branes on $\mathbb{C}^{4} /\left(\mathbb{Z}_{2} \times \mathbb{Z}_{2} \times \mathbb{Z}_{2}\right)$ parallels the analysis of D3-branes on $\mathbb{C}^{3} /\left(\mathbb{Z}_{2} \times \mathbb{Z}_{2}\right)$. The theory of D1branes on the singular orbifold is an $\mathbf{N}=(0,2)$ super Yang-Mills theory in two dimensions. In the absence of discrete torsion the singularity in the moduli space is resolved by introducing FayetIliopoulos terms in the action of the super Yang-Mills theory. The moduli space with perturbed Dflatness conditions, implementing the resolution of the orbifold, can be studied using the paraphernalia of toric geometry, where the monomials of the toric description are provided by the unperturbed Fflatness condition of the theory. In the presence of discrete torsion, however, it is the F-term of the $(0,2)$ theory that admits a perturbation, involving six parameters. The D-flatness conditions, however, remain unaltered with respect to the theory on the singular orbifold. This corresponds to a deformation of the singularity, rather than its resolution. The perturbation of the F-flatness conditions prevents employment of toric geometry in the description of the deformed variety. One is led to consider the gauge-invariant quantities to furnish a description of the deformed variety. It is found that, after desingularisation by F-term perturbations, the variety describing the moduli space of the gauge theory retains a stable singularity of codimension three (line singularity), in the same manner as its three-dimensional counterpart retains a conifold singularity after deformations [ 4 , 5].

The plan of the article is as follows. We recount some features of the projective representations of $\mathbb{Z}_{2} \times \mathbb{Z}_{2} \times \mathbb{Z}_{2}$ in $\S$. In $\oint_{3}$ we study the twisted sector of closed string theory on $\mathbb{C}^{4} /\left(\mathbb{Z}_{2} \times \mathbb{Z}_{2} \times \mathbb{Z}_{2}\right)$ in the presence of discrete torsion. This analysis enables us to determine the number of perturbation parameters allowed in the gauge theory. The low energy effective gauge theory of the D-brane on the orbifold is discussed in the $\S$. In $\S[$ we consider the vacua of the resulting gauge theory and find out the corresponding moduli spaces both with and without deformations, before concluding in $\S$.

NOTATIONS AND CONVENTIONS: Unless explicitly declared otherwise, we follow the following conventions in notation and terminology in the sequel.

- The terms resolution (or blow up) and deformation of singularities are used in the usual senses. The term desingularisation is used generally to mean either of these two, therby encompassing partial removal of singularities. 
- For subscripts, uppercase letters from the middle of the alphabet, e.g. $I, J, K$, etc. assume values in $\{1,2,3,4\}$, while the corresponding lower-case letters, namely, $i, j, k$ etc., assume values in $\{1,2,3\}$.

- The Pauli matrices $\sigma_{I}$ are chosen to be the following:

$$
\sigma_{1}=\left(\begin{array}{cc}
0 & 1 \\
1 & 0
\end{array}\right) \quad \sigma_{2}=\left(\begin{array}{cc}
0 & -i \\
i & 0
\end{array}\right) \quad \sigma_{3}=\left(\begin{array}{cc}
1 & 0 \\
0 & -1
\end{array}\right) \quad \sigma_{4}=\left(\begin{array}{ll}
1 & 0 \\
0 & 1
\end{array}\right)
$$

- No sum is intended on repeated indices.

\section{Projective representation \& discrete torsion}

In considering desingularisations of orbifolds of the type $\mathbb{C}^{4} / G$, discrete torsion corresponds to nontrivial elements of the second cohomology group of the finite group $G$. It is incorporated in the theory through the action of the group $G$ on the Chan-Paton degrees of freedom by using an adjoint action by a projective representation of $G$. In this section we will collect some facts [15] about the projective representation of $G$, relevant for the present article.

Given a finite group $G$, a mapping $\rho: G \longrightarrow G L(n, \mathbb{C})$ is called a projective $\alpha$-representation of $G$ (over the field $\mathbb{C}$ ), provided there exists a mapping $\alpha: G \times G \longrightarrow U(1)$, such that

1. $\rho(g) \rho\left(g^{\prime}\right)=\alpha\left(g, g^{\prime}\right) \rho\left(g g^{\prime}\right)$,

2. $\rho(\mathbf{1})=\mathbb{I}_{n}$,

for all elements $g, g^{\prime} \in G$, where 1 denotes the identity element of $G$ and $\mathbb{I}_{n}$ denotes the $n \times n$ identity matrix in $G L(n, \mathbb{C})$. Let us note that one can define a projective representation over more general fields [15]. Here we are referring to the projective matrix representation over the field of complex numbers as the projective representation. It can be shown that $\alpha$ is a $U(1)$-valued two-cocycle of the second cohomology group $H^{2}(G, U(1))$ of the finite group $G$. For our purposes $\alpha$ will be a complex number with unit modulus.

Generally, the second cohomology group $H^{2}(G, U(1))$ of a direct-product group of the form $G=\bigotimes_{1}^{m} \mathbb{Z}_{n}$, is isomorphic to $\bigotimes_{1}^{m(m-1) / 2} \mathbb{Z}_{n}$. Let $\mathfrak{g}_{i}$ denote the generator of the $i$-th $\mathbb{Z}_{n}$ factor appearing in $G$, i.e. $G=\bigotimes_{1}^{m}\left\langle\mathfrak{g}_{i}\right\rangle$. Let $g_{i}, i=1,2, \cdots, m$, denote the generators of $G$. Let us also define

$$
\mu(i)=\prod_{a=1}^{n-1} \alpha\left(g_{i}^{a}, g_{i}\right) \quad \text { and } \quad \beta(i, j)=\alpha\left(g_{i}, g_{j}\right) \alpha\left(g_{j}, g_{i}\right)^{-1}, \quad i, j=1,2, \cdots, m .
$$

\footnotetext{
${ }^{1}$ See, however, [14] for more rigorous considerations on discrete torsion.

${ }^{2}$ Usually the range of the map $\alpha$ is taken to be the multiplicative group $\mathbb{C}^{\star}$ of the field $\mathbb{C}$ of complex numbers. Here we will only consider the map $\alpha$ with unit modulus. So, we have taken the range to be $U(1)$.
} 
We may set $\beta$ to be an $n$-th root of unity and $\mu=1$, by replacing $\alpha$, if necessary, by a cohomologous cocycle. The corresponding projective $\alpha$-representation of $G$ is given in [15] for special values of $\beta$. For our purposes it suffices to quote the results for the special case with $m=3$ and $n=2$.

Thus, we will consider the projective representations of the group $G=\mathbb{Z}_{2} \times \mathbb{Z}_{2} \times \mathbb{Z}_{2}$. Let us assume that $G$ has the following action on the four coordinates $Z_{1}, Z_{2}, Z_{3}, Z_{4}$ of $\mathbb{C}^{4}$ :

$$
\begin{aligned}
& g_{1}:\left(Z_{1}, Z_{2}, Z_{3}, Z_{4}\right) \longmapsto\left(-Z_{1},-Z_{2}, Z_{3}, Z_{4}\right), \\
& g_{2}:\left(Z_{1}, Z_{2}, Z_{3}, Z_{4}\right) \longmapsto\left(-Z_{1}, Z_{2},-Z_{3}, Z_{4}\right), \\
& g_{3}:\left(Z_{1}, Z_{2}, Z_{3}, Z_{4}\right) \longmapsto\left(-Z_{1}, Z_{2}, Z_{3},-Z_{4}\right) .
\end{aligned}
$$

Let $\mathfrak{g}_{1}, \mathfrak{g}_{2}$ and $\mathfrak{g}_{3}$ denote the three generators of the three $\mathbb{Z}_{2}$ factors. A generic element of $G$ can be written as $g=\mathfrak{g}_{1}^{a} \mathfrak{g}_{2}^{b} \mathfrak{g}_{3}^{c}$. We will denote this element by the symbol $(a b c)$. Choosing the action of each of the $\mathbb{Z}_{2}$ factors to be a change of sign of $Z_{1}$ and one more out of $Z_{2}, Z_{3}$ and $Z_{4}$, we can write $g_{i}$ from (2.2) as

$$
\begin{aligned}
& g_{1}=\mathfrak{g}_{1}=(100), \\
& g_{2}=\mathfrak{g}_{2}=(010), \\
& g_{3}=\mathfrak{g}_{3}=(001) .
\end{aligned}
$$

The second cohomology group $H^{2}(G, U(1))$ of $G$ is isomorphic to $\mathbb{Z}_{2} \times \mathbb{Z}_{2} \times \mathbb{Z}_{2}$ [15]. The three generators of the latter may be taken to be

$$
\alpha_{1}\left((a b c),\left(a^{\prime} b^{\prime} c^{\prime}\right)\right)=i^{\left(a b^{\prime}-b a^{\prime}\right)}, \quad \alpha_{2}\left((a b c),\left(a^{\prime} b^{\prime} c^{\prime}\right)\right)=i^{\left(b c^{\prime}-c b^{\prime}\right)}, \quad \alpha_{3}\left((a b c),\left(a^{\prime} b^{\prime} c^{\prime}\right)\right)=i^{\left(c a^{\prime}-a c^{\prime}\right)} .
$$

Let us note that $\alpha_{i}((a b c),(a b c))=1$ for $i=1,2,3$. Hence, by (2.1), we have $\mu(i)=1$, for $i=1,2,3$. In what follows we will consider the element $\alpha=\alpha_{1} \alpha_{2} \alpha_{3}$. Thus, we have, for all $g, g^{\prime} \in G$,

$$
\begin{aligned}
\alpha\left(g, g^{\prime}\right)=i \quad \text { if } g \neq g^{\prime}, \\
=1 \quad \text { if } g=g^{\prime},
\end{aligned}
$$

and consequently, $\mu(i)=1$ for $i=1,2,3$, and $\beta=-1$. There are two irreducible $\alpha$-representations of $G$, which are not linearly equivalent. These are given by [15]

$$
\rho\left(g_{i}\right)= \pm \sigma_{i}
$$

The discrete torsion appearing in the path integral is determined by the choice of the two-cocycle. For example, for the above choice of the two-cocycle, namely $\alpha=\alpha_{1} \alpha_{2} \alpha_{3}$, the discrete torsion is given by [4, 5]

$$
\begin{aligned}
\varepsilon\left((a b c),\left(a^{\prime} b^{\prime} c^{\prime}\right)\right) & =\left(\alpha\left((a b c),\left(a^{\prime} b^{\prime} c^{\prime}\right)\right)\right)^{2}, \quad(a b c) \neq\left(a^{\prime} b^{\prime} c^{\prime}\right) \\
& =(-1)^{a b^{\prime}-b a^{\prime}+b c^{\prime}-c b^{\prime}+c a^{\prime}-a c^{\prime}},
\end{aligned}
$$


which is the form used in [16]. Each given value of the discrete torsion corresponds to a variety with a different topology and leads to different types of gauge theories. These different varieties are related by mirror symmetry [16].

Let us point out that the discrete torsion $\alpha$ used in (2.5) is by no means more general than any other two-cocycle. This can be interpreted as a phase between two of the $\mathbb{Z}_{2}$ factors in $G$ by a change of basis. This fact will be reflected in the moduli space of the brane in that, the maximally deformed moduli space retains a singular line, unlike the point in [甘, 5].

\section{String(ent) restrictions on deformations}

It has been known that string theory can be defined on certain kinds of singular spaces, especially on orbifolds. In sigma-model compactification on orbifolds, the spectrum of string theory receives contributions from the twisted sectors, thereby rendering string theory well-defined on such spaces. In considering D-branes on orbifolds, the orbifold is realised as the moduli space of the gauge theory on the world-volume of the brane. Resolution or deformation of the quotient singularity is effected by perturbing the gauge theory. However, compatibility of the desingularised D-brane moduli space with string theory imposes stringent restrictions on such extra terms. In this section, we discuss these restrictions.

The four-dimensional orbifold $\mathbb{C}^{4} /\left(\mathbb{Z}_{2} \times \mathbb{Z}_{2} \times \mathbb{Z}_{2}\right)$ in the blown down limit may be defined as an affine variety embedded in $\mathbb{C}^{5}$ by the polynomial equation $\mathcal{F}(x, y, z, w, t)=0$, where the polynomial is $\mathcal{F}(x, y, z, w, t)=x y z w-t^{2}$, and $x, y, z, w, t$ are the coordinates of $\mathbb{C}^{5}$. The $\mathbb{Z}_{2} \times \mathbb{Z}_{2} \times \mathbb{Z}_{2}$ symmetry can be made conspicuous by expressing the coordinates of $\mathbb{C}^{5}$ in terms of the affine coordinates of the covering space of the variety, namely, $\mathbb{C}^{4}$. Explicitly, $x=u_{1}^{2}, y=u_{2}^{2}, z=u_{3}^{2}, w=u_{4}^{2}, t=u_{1} u_{2} u_{3} u_{4}$, with $\left\{u_{1}, u_{2}, u_{3}, u_{4}\right\} \in \mathbb{C}^{4}$. The polynomial $\mathcal{F}$ remains invariant under the three independent transformations, which change the signs of, say $u_{1}$, together with one out of $\left\{u_{I} \mid I=2,3,4\right\}$ in turn.

Let us briefly recount the algebraic deformations of the equation $\mathcal{F}=0$. The possible deformations are given by the ring of polynomials $Q=\mathbb{C}[x, y, z, w, t] /\langle\partial \mathcal{F}\rangle$, where $\partial \mathcal{F}$ stands for the set of the partial derivatives of $\mathcal{F}$ with respect to each of the arguments, i.e.

$$
\partial \mathcal{F}=\{\partial \mathcal{F} / \partial x, \partial \mathcal{F} / \partial y, \partial \mathcal{F} / \partial z, \partial \mathcal{F} / \partial w, \partial \mathcal{F} / \partial t\},
$$

and $\langle\cdots\rangle$ denotes the ideal generated by $\cdots$. Thus, we have

$$
\begin{aligned}
\langle\partial \mathcal{F}\rangle & =\langle x y z, y z w, x y w, x z w, t\rangle, \\
Q & =\left\langle 1, x^{a}, y^{b}, z^{c}, w^{d}, x y, x z, x w, y z, y w, z w\right\rangle,
\end{aligned}
$$

where $a, b, c, d$ are arbitrary integers. Among the generators of $Q$, terms such as $x y$ deform the six fixed planes such as $z w=0$, terms like $x$ deform the four fixed lines which correspond to codimension-three singularities and finally 1 deforms the double-point singularity with codimension four at the origin, $t^{2}+x^{2}+y^{2}+z^{2}+w^{2}=0$. 
However, as mentioned before, a physical theory, as the one we are considering, is not necessarily potent enough to contain all the deformations that are mathematically admissible. Within the scope of our discussion with D1-branes, the four-dimensional orbifold is realised as the moduli space of an $\mathbf{N}=(0,2)$ super-Yang-Mills theory and its deformations are subject to consistency requirements imposed by string theory. Considering branes in the closed or type-II string theory, these consistency conditions are determined by the twisted sector of the theory on the orbifold. Of the generators of the ring $Q$, only those deformations are physically allowed, that correspond to marginal operators in the closed string theory on the orbifold. The marginal operators are related by supersymmetry to the Ramond-Ramond (RR) ground states of the string theory. The latter, in turn, are determined by the cohomology of the smooth target space that limits to the orbifold under consideration. To be more explicit, let $X$ be a manifold, and let $X^{\prime}=X / G$ be an orbifold, where $G$ is a finite group of order $|G|$. Let $\tilde{X}$ be a desingularisation of the orbifold $X^{\prime}$.

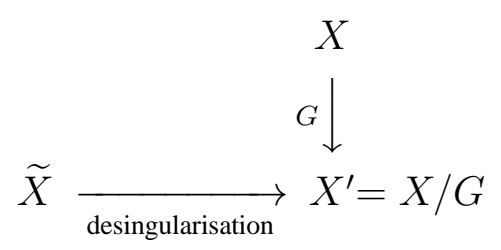

Considering string theory on the orbifold $X^{\prime}$, the above-mentioned computation of RR ground states yields the cohomology $H^{\star}(\widetilde{X})$ of $\widetilde{X}$.

The desingularisation $\widetilde{X} \longrightarrow X^{\prime}$ can be effected in two different ways. One way is to blow up the singularity at the origin. This corresponds to turning on a Fayet-Iliopoulos term in the $\mathbf{N}=(0,2)$ gauge theory, and thereby perturbing the D-term of the gauge theory [13]. The other way is to deform the singularity, discussed above. This corresponds to perturbing the F-flatness conditions in the gauge theory and is relevant for us in considering orbifolds with discrete torsion. At any rate, in order to count the physically admissible perturbation modes in the gauge theory we need to consider closed strings on the orbifold and evaluate the cohomology.

Let us compute the cohomology of the space $\widetilde{X}$ for this case, following [2, 5, 16, 17]. The general strategy is as follows. Given an element $g$ of the group $G$, we first find out the subset of $X$ that is fixed under $g$. Let us denote this subset by $X_{g}$. The subset $X_{g}$ is endowed with $(p, q)$-forms, denoted $\omega_{g}^{p, q}$. Let $\Omega_{g}^{p, q}$ denote the set of $(p, q)$-forms on $X_{g}$ and let $\widetilde{\Omega}^{p, q}$ denote the set of all $(p, q)$-forms on $\tilde{X}$. The $(p, q)$-forms $\omega_{g}^{p, q} \in \Omega_{g}^{p, q}$, which are invariant under the group $G$, that is, which satisfy

$$
\varepsilon(h, g) R(h) \omega_{g}^{p, q}=\omega_{g}^{p, q}, \quad \omega_{g}^{p, q} \in \Omega_{g}^{p, q}, \quad h \in G
$$

contribute to $\widetilde{\Omega}^{p+s, q+s}$, where $s$ is the age of $g \in G$, defined by $s=\sum_{I=1}^{4} \vartheta_{I}$, if $g: Z_{I} \longmapsto e^{2 \pi i \vartheta_{I}} Z_{I}$, where $Z_{I}, I=1,2,3,4$ are the coordinates of $X$. Here $R(h)$ denotes some representation of the element $h \in G$ and $\varepsilon$ is the discrete torsion defined in (2.7). The cohomology in absence of discrete torsion can be obtained by setting $\varepsilon=1$. 
For the case at hand, the different elements of the group $G=\mathbb{Z}_{2} \times \mathbb{Z}_{2} \times \mathbb{Z}_{2}$ fixes three kinds of subsets in $X=\mathbb{C}^{4}-$ contributing $h^{p q}(p, q)$-forms to $\widetilde{\Omega}^{p, q}$. First, the identity of $G$ fixes $\mathbb{C}^{4}$ itself, that is, $X_{(000)}=\mathbb{C}^{4}$. We shall refer to the corresponding contribution to the cohomology as the contribution from the untwisted sector. The $G$-invariant forms are $1, d Z_{I} \wedge d \bar{Z}_{I}$ and $d Z_{1} \wedge d Z_{2} \wedge d Z_{3} \wedge$ $d Z_{4}$ and some of the $d Z$ 's replaced by their complex conjugates. The contribution to the cohomology $H^{\star}(\widetilde{X})$ is summarised in the following Hodge diamond:

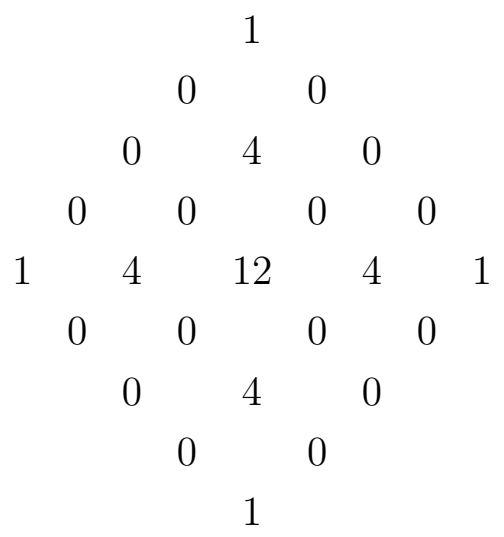

These elements of $H^{\star}(\widetilde{X})$ need be supplemented with the contribution from the twisted sector, that corresponds to the other two kinds of fixed sets. These are contributions from the $G$-invariant forms from the fixed subsets of $X$, under the non-trivial elements of $G$. We will refer to these as the contribution from the twisted sector. The element $(111) \in G$ fixes a point, $X_{(111)}=\{0\}$, the origin of $\mathbb{C}^{4}$, while each of the other six elements of $G$ leaves fixed a set $\mathbb{C}^{2} \subset X$.

The contribution from the untwisted sector is the same both in the presence and absence of discrete torsion. Contribution from the twisted sectors are different in the two cases. Let us consider both the cases in turn.

- Without discrete torsion:

In the absence of discrete torsion the condition of $G$-invariance of the $(p, q)$-forms is given by (3.3) with $\varepsilon=1$.

Only the $(0,0)$-form 1 is defined on $X_{(111)}$, which is a point. This is obviously $G$-invariant. The age of $(111) \in G$ is $s=2$. Thus, this form contributes to $\widetilde{\Omega}^{2,2}$, with $h^{22}=1$.

Each of the other six elements of $G$ has age $s=1$. Each fixes a $\mathbb{C}^{2} \subset X$. For example, (100) fixes the $\mathbb{C}^{2}$ coordinatised by $Z_{3}$ and $Z_{4}$. The $G$-invariant forms on this $\mathbb{C}^{2}$ are $1, d Z_{i} \wedge d \bar{Z}_{i}$, $i=3,4$ and $d Z_{3} \wedge d Z_{4} \wedge d \bar{Z}_{3} \wedge d \bar{Z}_{4}$. Taking into account the shift by the age of this element, the contribution to the Hodge numbers are: $h^{11}=1, h^{22}=2, h^{33}=1$. Similar consideration with each of the other five elements leads to similar contribution to the cohomology. The total 
contribution from the twisted sector to $H^{\star}(\widetilde{X})$ is summarised in the following Hodge diamond:

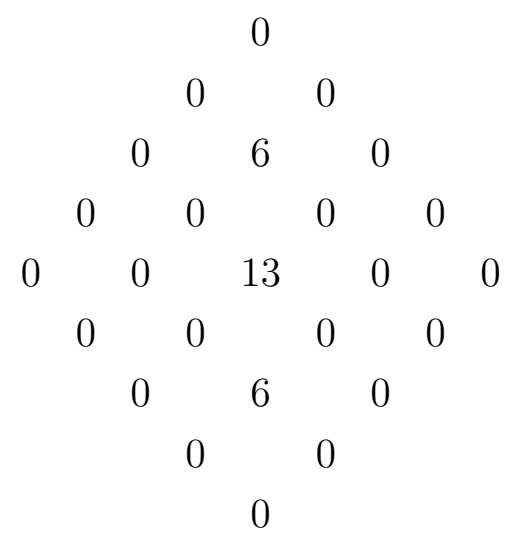

Let us point out that since the contribution from the twisted sector is $h^{11}=6$, the number of perturbation parameters allowed in the gauge theory in the absence of discrete torsion is 6 . This is in keeping with the fact that there are six deformations of the D-flatness condition [13]. Let us point out that there is no symmetry guaranteeing that the contributions from the six sectors mentioned above should be the same. That the contributions are indeed the same is a coincidence and has to be checked on a case by case basis for each of the elements.

- With discrete torsion:

In the presence of discrete torsion, the condition of $G$-invariance of forms is generalised to (3.3), with $\varepsilon$ defined by (2.7).

The contribution to $H^{\star}(\widetilde{X})$ corresponding to (111) happens to remain the same as that in the case without discrete torsion, namely $h^{22}=1$.

However, the contribution from the other six do differ. Considering (100), again, the invariant forms on the fixed $\mathbb{C}^{2}$ are $d Z_{3} \wedge d Z_{4}, d \bar{Z}_{3} \wedge d \bar{Z}_{4}, d Z_{3} \wedge d \bar{Z}_{4}$ and $d \bar{Z}_{3} \wedge d Z_{4}$. Thus, taking into account the shift by the age $s=1$ of each of the elements, the contribution to $H^{\star}(\widetilde{X})$ is $h^{22}=2, h^{31}=1$. Analysing similarly the contributions from the other five elements, we get, in the long run, the following Hodge diamond arising from the twisted sector in the presence of discrete torsion:

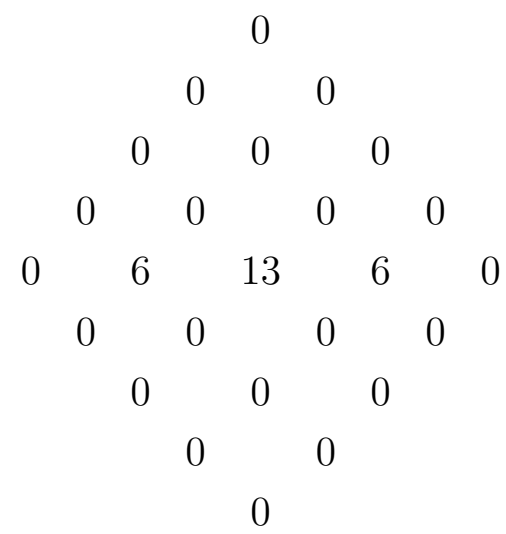


Thus, we may have at most six deformations for the F-term, as they correspond to deformation of the orbifold singularity and determined by $h^{31}$. In the next section we will find out the six possible terms.

Let us note that the element $(111) \in G$ has age $s=2$ and leads to a terminal singularity not giving in to a crepant resolution. However, this does not affect the present analysis, as this is confined to a consideration of perturbation of the gauge theory by the six parameters that correspond to the six $(3,1)$-forms, none of which have been contributed by (111). Let us point out in passing that by turning on the discrete torsion we get the $h^{11}$ and $h^{(4-1) 1}=h^{31}$ interchanged. This signifies that the resulting manifolds are related by mirror symmetry.

\section{The gauge theory of the branes}

In the regime of weak string coupling, D-branes admit a description in terms of a supersymmetric Yang-Mills theory (SYM) on their world volumes. The moduli space of the SYM is interpreted as the space-time. Referring to the case at hand, the world-volume theory of $n$ coalescing D1-branes in type-IIB theory is taken to be the dimensional reduction of the $\mathrm{N}=1, \mathrm{D}=10 U(n) \mathrm{SYM}$ on $\mathbb{C}^{4}$, or equivalently, the reduction of the $N=4, D=4 S Y M$ on $\mathbb{C}^{2}$, down to two dimensions. The resulting theory is an $\mathrm{N}=(8,8), \mathrm{D}=2 U(n) \mathrm{SYM}$. We will consider a fourfold transverse to the world-volume of the D1-branes obtained as an orbifold of $\mathbb{C}^{4}$. The D1-brane is taken to be lying along the 9-th direction, evolving in time along the 0 -th direction. The $\mathbb{C}^{4}$ is coordinatised by $Z_{I}$, $I=1,2,3,4$. Let $G$ be a finite group of order $|G|$. In order to retain some supersymmetry, $G$ must be a subgroup of the holonomy group of the fourfold, namely $S U(4)$. The theory of a single D1-brane on the blown down orbifold $\mathbb{C}^{4} / G$ is obtained by starting with a theory of $|G|$ coalescing D1-branes in two dimensions and then quotienting the resulting gauge theory with gauge group $U(|G|)$, by $G$. The resulting theory turns out to have $\mathrm{N}=(0,2)$ supersymmetry in two dimensions [18].

It is convenient, in practice, to start with the $\mathrm{N}=1, \mathrm{D}=10$ gauge theory reduced to two dimensions written in a $\mathbf{N}=(0,2)$ notation [19, 20]. Finally one substitutes the fields those have survived the orbifold projection. Through the projection the supersymmetry will get broken down to $(0,2)$. This theory corresponds to the theory of a D1-brane on the singular orbifold $\mathbb{C}^{4} / G$. The deformation and/or blow up of the orbifold $\mathbb{C}^{4} / G$ corresponds to adding extra terms to the abovementioned $(0,2)$ action, with all fields taken to be the ones surviving the projection [18].

\subsection{The gauge theory: before projection}

Let us begin with an inventory of the multiplets of $\mathrm{N}=(0,2), \mathrm{D}=2$ super-Yang-Mills theory $[18,20,21]$. The field-content of the theory is as follows. There are four complex bosonic fields, denoted by $Z_{I}, I=1,2,3,4$, identified with the four complex dimensions transverse to the world volume. There are eight left-handed and eight right-handed Majorana-Weyl spinors which constitute 
four left-handed Dirac fermions and four right-handed Dirac fermions. The left handed fermions are $\lambda_{-}$satisfying $\left(\lambda_{-}\right)^{\dagger}=\bar{\lambda}_{-}$and the three other can be grouped together as $\lambda_{I J}$ which is antisymmetric in $I J$ and satisfies $\left(\lambda_{I J}\right)^{\dagger}=\epsilon_{I J K L} \lambda_{K L}$. Finally, there is a vector field, whose two components will be denoted by $v_{ \pm}$, in the light-cone coordinates.

The fields mentioned above may be assorted into three supersymmetry multiplets, that is, into three superfields. The vector field and the fermion $\lambda_{-}$, from the left sector are combined to form the vector multiplet, whose components in the Wess-Zumino gauge are written as,

$$
\begin{aligned}
& A_{-}=v_{-}-2 i\left(\theta \bar{\lambda}_{-}+\bar{\theta} \lambda_{-}\right)+2(\theta \bar{\theta}) D, \\
& A_{+}=(\theta \bar{\theta}) v_{+},
\end{aligned}
$$

where $v_{ \pm}$are the vector fields and $D$ is an auxiliary field. The corresponding field strength is given by

$$
F=\lambda_{-}-\theta\left(F_{-+}+i D\right)+2 i(\theta \bar{\theta}) \partial_{+} \lambda_{-}
$$

where $F_{-+}=\partial_{-} v_{+}-\partial_{+} v_{-}$.

Four bosonic chiral multiplets are formed from $Z_{I}$ and four Dirac spinors $\psi_{I}$ coming from right sector. The corresponding superfield takes the form

$$
\phi_{I}=Z_{I}+\sqrt{2} \theta \psi_{I}+2 i \bar{\theta} \theta \nabla_{+} Z_{I}
$$

where we have defined $\nabla_{ \pm}=\partial_{ \pm}-i q A_{ \pm}$, and $q$ denotes the charge of the vector multiplet under the gauge group $U(N)$.

Finally, the three fermions $\lambda^{I J}$, with hermitian conjugates defined as $\left(\lambda^{I J}\right)^{\dagger}=\epsilon^{I J K L} \lambda^{K L}$, are gathered into three fermionic chiral multiplets which assume the following form:

$$
\Lambda_{i 4}=\lambda_{i 4}-\sqrt{2} \theta G_{i 4}-2 i(\bar{\theta} \theta) \nabla_{+} \lambda_{i 4}-\sqrt{2} \bar{\theta} E_{i} ; \quad i=1,2,3
$$

where $G_{i 4}$ denotes a bosonic auxiliary field and $E_{i}$ represents a bosonic chiral field.

The contributions of these multiplets to the action of the theory can be obtained [20] from the reduction of ten-dimensional action. A more convenient way would be to start with four-dimensional $\mathrm{N}=1$ action reduced to two dimensions which is a $(2,2)$ theory and then by integrating out one of the $\theta$ 's. These result in fixing the chiral fields $E_{i}$ as $E_{i}=\left[\phi_{i}, \phi_{4}\right]$. The contributions of the abovementioned multiplets to the Lagrangian of the theory are given by,

$$
\begin{aligned}
L_{A} & =\frac{1}{2 e^{2}} \int d \theta d \bar{\theta} \operatorname{Tr}(\bar{F} F) \\
L_{\phi} & =i \sum_{I} \int d \theta d \bar{\theta} \operatorname{Tr}\left(\bar{\phi}_{I} \nabla_{-} \phi_{I}\right) \\
L_{\Lambda} & =\frac{1}{2} \sum_{i} \int d \theta d \bar{\theta} \operatorname{Tr}\left(\bar{\Lambda}_{i 4} \Lambda_{i 4 i}\right)
\end{aligned}
$$


The total Lagrangian obtained as the sum of the three pieces (4.5) admits a superpotential term, while retaining $\mathbf{N}=(0,2)$ supersymmetry. The corresponding piece of the Lagrangian is given by

$$
L_{\mathcal{W}}=\frac{1}{\sqrt{2}} \int d \theta \mathcal{W}+\text { h.c., }
$$

where $\mathcal{W}$ is a chiral fermionic field, the superpotential. The general form of $\mathcal{W}$ is $\mathcal{W}=\operatorname{Tr} \sum_{i} \Lambda_{i 4} J^{i}$, where $J^{i}$ denotes a bosonic chiral field satisfying the supersymmetry-constraint

$$
\sum_{i} E_{i} J^{i}=0
$$

Thus, to sum up, the total action is given by

$$
L=L_{A}+L_{\phi}+L_{\Lambda}+L_{\mathcal{W}}
$$

The reduced two-dimensional theory, in absence of extra couplings, the chiral field $J^{i}$ in $\mathcal{W}$ assumes the form, $J^{i}=\sum_{j, k} \epsilon^{i j k}\left[\phi_{j}, \phi_{k}\right]$, which satisfies the supersymmetry constraint (4.7), thanks to the Jacobi identity.

The action corresponding to (4.8) has a global $U(1)^{4}$ symmetry associated with the phases of the bosonic fields of which the global $U(1)$ is an R-symmetry of the theory. The bosonic potentials, known as the F-term and the D-term, are obtained by integrating out the auxiliary fields $G_{i 4}$ and $D$, respectively, appearing in $L$. These are given by

$$
\begin{aligned}
& U_{F}=2 \sum_{I, J} \operatorname{Tr}\left[Z_{I}, Z_{J}\right]^{2}, \\
& U_{D}=2 e^{2} \sum_{I} \operatorname{Tr}\left[Z_{I}, \bar{Z}_{I}\right]
\end{aligned}
$$

respectively.

As we will see in presence of the coupling of D-string to twisted sector modes the superpotential as well as the form the chiral fields $E_{i}$ will get modified.

\subsection{The gauge theory: after projection}

Having discussed some generalities let us now implement the projection by $G=\mathbb{Z}_{2} \times \mathbb{Z}_{2} \times \mathbb{Z}_{2}$. The order of $G$ is $|G|=8$. Hence we start with 8 branes at the origin, choosing the gauge group to be $U(8)$, and then quotient the theory by $G$. As mentioned earlier, the action of the group on the ChanPaton indices is given by the regular representation, obtained as direct sum of two copies of each of the projective representations given in (2.6). Thus, we have,

$$
r\left(g_{i}\right)=\operatorname{Diag}\left\{\sigma_{i}, \sigma_{i},-\sigma_{i},-\sigma_{i}\right\}
$$


where $r\left(g_{i}\right)$ denotes the regular representation of the generator $g_{i}$ of the group $G$. The action of the generators $g_{i}$ on the Lorentz indices is as given in (2.2).

The projections are given by adjoint action of the regular representations $r\left(g_{i}\right)$ on the supermultiplets. In terms of the respective superfields, they take the form,

$$
\begin{aligned}
& r\left(g_{i}\right) A_{\mu} r\left(g_{i}\right)^{-1}=A_{\mu}, \\
& r\left(g_{i}\right) Z_{I} r\left(g_{i}\right)^{-1}=\sum_{I=1}^{4} R\left(g_{i}\right)_{I J} Z_{J}, \\
& r\left(g_{i}\right) \lambda_{I J} r\left(g_{i}\right)^{-1}=\sum_{I^{\prime}, J^{\prime}=1}^{4} R\left(g_{i}\right)_{I I^{\prime}} R\left(g_{i}\right)_{J J^{\prime}} \lambda_{I^{\prime} J^{\prime}},
\end{aligned}
$$

where we have introduced three matrices $R\left(g_{i}\right)_{I J}$, such that the equations (2.2) read

$$
g_{i}: Z_{I} \longmapsto \sum_{J=1}^{4} R\left(g_{i}\right)_{I J} Z_{J}
$$

for $I=1,2,3,4$ and $i=1,2,3$.

The gauge group of the theory after projections (4.12) breaks down to $U(2) \times U(2)$ of which the center of mass $U(1)$ decouples which plays the role of the unbroken $U(1)$ for the single brane. The rest of the group $U(1) \times S U(2) \times S U(2)$ is broken by the vacuum expectation values of the following Higgs field

$$
Z_{I}=\left(\begin{array}{cc}
0 & z_{I} \times \sigma_{I} \\
w_{I} \times \sigma_{I} & 0
\end{array}\right),
$$

where $z_{I}, w_{I}$ are $2 \times 2$ matrices. The charges are assigned through,

$$
z_{I} \longmapsto U z_{I} V^{\dagger}, \quad w_{I} \longmapsto V w_{I} U^{\dagger}
$$

where $U$ and $V$ belong to the two $S U(2)$ 's and $z_{I}$ and $w_{I}$ have opposite charges under the relative $U(1)$. The representation of the chiral Fermi field is the same as that of the commutators of the bosonic fields, which is consistent with the presence of the $E$ field in the multiplet. Thus, we have

$$
\Lambda_{i 4}=\left(\begin{array}{cc}
\lambda_{i}^{1} \times \sigma_{i} & 0 \\
0 & \lambda_{i}^{2} \times \sigma_{i}
\end{array}\right) .
$$

The fields after projection are then substituted in the action in order to derive the theory of the single brane on $\mathbb{C}^{4} / G$. The moduli space is described by the solutions of the conditions of F-flatness and D-flatness, obtained by minimising the F-term and the D-term, respectively. The F-flatness conditions are given by

$$
\begin{array}{ll}
z_{j} w_{i}+z_{i} w_{j}=0, & z_{4} w_{i}-z_{i} w_{4}=0, \\
w_{j} z_{i}+w_{i} z_{j}=0, & w_{4} z_{i}-w_{i} z_{4}=0,
\end{array}
$$


for $i=1,2,3$.

Let us now consider the deformation of this moduli space in the presence of non-zero coupling with fields in the twisted sector of the closed string theory. In the discussion of the closed string twisted sectors in $\S 3$, we noticed that each of the six group elements which flips the sign of $Z_{I}$ 's pairwise contributes $(3,1)$, responsible for the deformation of the complex structure of the singular orbifold. These are complex numbers and hence couple naturally to the superpotential [4], 5]. Geometrically, these deform away codimension-two singularities.

One set of the natural modification of the superpotential comes from its four-dimensional analogue. Starting with a term of the form $\int d \theta \xi_{i} \phi_{i}$, where $\phi_{i}$ is a four-dimensional chiral field, reducing to two dimension and integrating one $\theta$ out yields a term

$$
\delta W=\sum_{i=1}^{3} \int d \theta^{+} \operatorname{Tr}\left(\boldsymbol{\xi}_{i} \Lambda_{i 4}\right)
$$

where $\boldsymbol{\xi}_{i}$ denotes the coupling parameters, of the form

$$
\boldsymbol{\xi}_{i}=\left(\begin{array}{cc}
\xi_{i} \times \sigma_{i} & 0 \\
0 & \xi_{i} \times \sigma_{i}
\end{array}\right) .
$$

The form of $\boldsymbol{\xi}_{i}$ is determined by the gauge-invariance of the coupling and by the fact that its introduction does not break supersymmetry according to (4.7). A simple calculation leads to the above expression without any loss of generality, provided $\boldsymbol{\xi}_{i}$ do not depend on the other fields.

These perturbations give rise to a deformation of the F-term equation as

$$
\begin{aligned}
z_{j} w_{i}+z_{i} w_{j} & =e_{i j k} \xi_{k}, \\
w_{j} z_{i}+w_{i} z_{j} & =e_{i j k} \xi_{k},
\end{aligned}
$$

where $i=1,2,3$, and $e_{123}=+1, e_{i j k}$ is symmetric under interchange of $i, j$ but non zero only when $i, j, k$ are all different.

The form of the other perturbations can be obtained by treating all the 4 coordinates of the transverse space on same footing. This perturbations can be introduced by making use of the freedom in the definition of $E$ which correspond to a perturbation of the field $E_{i}$ in the fermionic multiplet given by

$$
E_{i} \rightarrow E_{i}+\boldsymbol{\eta}_{i}
$$

where

$$
\boldsymbol{\eta}_{i}=\left(\begin{array}{cc}
\eta_{i} \times \sigma_{i} & 0 \\
0 & \eta_{i} \times \sigma_{i}
\end{array}\right) .
$$

Here also the form of the coupling is determined by the fact that $\boldsymbol{\eta}_{i}$ should transform in a similar way as $E_{i}$ and it should not break supersymmetry (4.7). 
To sum up, the F-term equations following from these perturbations are given by

$$
\begin{array}{ll}
z_{j} w_{i}+z_{i} w_{j}=e_{i j k} \xi_{k}, & z_{4} w_{i}-z_{i} w_{4}=\eta_{i}, \\
w_{j} z_{i}+w_{i} z_{j}=e_{i j k} \xi_{i}, & w_{i} z_{4}-w_{4} z_{i}=\eta_{i} .
\end{array}
$$

We shall sometimes refer to (4.23) as the F-flatness equations. Let us note that, we have six parameters $\xi_{i}, \eta_{i}, i=1,2,3$, appearing in the perturbation of the gauge theory. This is in keeping with the fact that, the contribution to the cohomology of the resolved space, arising in the twisted sector is $h^{31}=6$, as discussed in $\S 3$.

\section{The moduli space and its deformation}

Let us now go over to finding the vacuum moduli space of the $(0,2)$ theory discussed in the previous section. The vacuum moduli space is the space of allowed values of the scalars $Z_{I}$, respecting the Fand D-flatness conditions, up to gauge equivalence.

\subsection{Gauge-invariant polynomials}

Thus, we have the four matrices $Z_{I}$, in the form (4.14), and the F-flatness conditions (4.23) on the non-vanishing $2 \times 2$ blocks of $Z_{I}$, namely $z_{I}$ and $w_{I}$. The equation of the variety describing the moduli space of the configuration will be written in terms of the gauge-invariant polynomials constructed out of $z_{I}$ and $w_{I}$. We proceed to describe these next.

Let us introduce the following expressions,

$$
\begin{aligned}
& \mathcal{P}_{I J K \cdots}=\frac{1}{2} \operatorname{Tr} z_{I} w_{J} z_{K} \cdots, \\
& \widetilde{\mathcal{P}}_{I J K \cdots}=\frac{1}{2} \operatorname{Tr} w_{I} z_{J} w_{K} \cdots,
\end{aligned}
$$

which we will refer to as polynomials. By the order of a polynomial, we mean the total number of $z$ and $w$ appearing in the polynomial - that is, the length of the word inside the trace. We need to introduce a few further notations which we list here:

$$
\begin{array}{r}
\mathcal{Q}_{I J}=z_{I} w_{J}, \quad \widetilde{\mathcal{Q}}_{I J}=w_{I} z_{J}, \\
x_{I}=\frac{1}{2} \operatorname{Tr} \mathcal{Q}_{I I}, \quad \widetilde{x}_{I}=\frac{1}{2} \operatorname{Tr} \widetilde{\mathcal{Q}}_{I I} .
\end{array}
$$

Let us also note that,

$$
\widetilde{x}_{I}=x_{I}
$$

It then follows from (5.1) that

$$
\mathcal{P}_{I J}=\frac{1}{2} \operatorname{Tr} \mathcal{Q}_{I J} \quad \widetilde{\mathcal{P}}_{I J}=\frac{1}{2} \operatorname{Tr} \widetilde{\mathcal{Q}}_{I J}
$$


Now, the polynomials of different orders defined by (5.1) are not linearly independent. We need to find out the independent polynomials in order to write down the equation of the variety. At this point let us note that from the gauge transformation (4.15) it follows that only the polynomials of even order are gauge-invariant quantities. Hence in what follows we shall not consider the polynomials of odd orders. Let us consider the remaining polynomials order by order.

- Order 2 polynomials

Using the constraints (4.23), it can be shown that $\mathcal{Q}_{I I}$ commute pairwise. Hence, assuming that these matrices are non-singular, the matrices $\mathcal{Q}_{I I}$ can be simultaneously diagonalised. From now on we assume that $\mathcal{Q}_{I I}, I=1,2,3,4$ are diagonal. Moreover, $\mathcal{Q}_{I J}$ with $I \neq J$ commute with $\mathcal{Q}_{I I}$, but not between themselves; for example, $\mathcal{Q}_{12}$ and $\mathcal{Q}_{23}$ do not commute. Hence, in a basis in which $\mathcal{Q}_{I I}$ are diagonal, $\mathcal{Q}_{I J}$, with $I \neq J$ are necessarily generic, i.e. not diagonal. It then follows, from the fact that $\mathcal{Q}_{I J}$, with $I \neq J$ and $\mathcal{Q}_{I I}$ commute, that $\mathcal{Q}_{I I}$ are all proportional to the two-dimensional identity matrix, $\mathbb{I}_{2}$. Moreover, if $z_{I}$ and $w_{I}$ are non-singular matrices, which also we assume, it follows that $\widetilde{\mathcal{Q}}_{I I}$ is also proportional to $\mathbb{I}_{2}$. Thus we have,

$$
\mathcal{Q}_{I I}=\widetilde{\mathcal{Q}}_{I I}=x_{I} \mathbb{I}_{2}
$$

and consequently,

$$
\mathcal{P}_{I I}=x_{I}
$$

The F-flatness conditions (4.23) impose further constraints on $\mathcal{Q}_{I J}$ with $I \neq J$. We will use these later.

Since the polynomials $\mathcal{P}_{I J}$ and $\widetilde{\mathcal{P}}_{I J}$ satisfy $\widetilde{\mathcal{P}}_{I J}=\mathcal{P}_{J I}$, it suffices to consider either set. The twelve polynomials $\mathcal{P}_{I J}$ with $I \neq J$ are not linearly independent. The F-flatness conditions (4.23) reduce these to a set of three independent ones. There are three further relations among these six, namely,

$$
\begin{aligned}
& \xi_{2} \mathcal{P}_{24}-\xi_{3} \mathcal{P}_{34}+\eta_{2} \mathcal{P}_{13}-\eta_{3} \mathcal{P}_{12}=0 \\
& \xi_{1} \mathcal{P}_{14}-\xi_{2} \mathcal{P}_{24}-\eta_{1} \mathcal{P}_{23}+\eta_{2} \mathcal{P}_{13}=\xi_{2} \eta_{2}-\xi_{1} \eta_{1} \\
& \xi_{1} \mathcal{P}_{14}-\xi_{3} \mathcal{P}_{34}+\eta_{1} \mathcal{P}_{23}+\eta_{3} \mathcal{P}_{12}=\xi_{3} \eta_{3}
\end{aligned}
$$

The equations (5.8) can be derived by considering certain polynomials of order 4 and using properties of trace of products of matrices and (4.23). For example, the first equation in (5.8) 
follows from $\mathcal{P}_{1243}$ as,

$$
\begin{aligned}
\mathcal{P}_{1243} & =\frac{1}{2} \operatorname{Tr} z_{1} w_{2} z_{3} w_{4} \\
& =\frac{1}{2} \operatorname{Tr}\left(\xi_{3}-z_{2} w_{1}\right)\left(z_{3} w_{4}+\eta_{3}\right) \\
& =\xi_{3} \mathcal{P}_{34}-\eta_{3} \mathcal{P}_{21}+\xi_{3} \eta_{3}-\widetilde{\mathcal{P}}_{1342} \\
\widetilde{\mathcal{P}}_{1342} & =\frac{1}{2} \operatorname{Tr} w_{1} z_{3} w_{4} z_{2} \\
& =\frac{1}{2} \operatorname{Tr}\left(\xi_{2}-w_{3} z_{1}\right)\left(-\eta_{2}+w_{2} z_{4}\right) \\
& =-\xi_{2} \eta_{2}+\eta_{2} \mathcal{P}_{13}+\xi_{2} \mathcal{P}_{42}-\mathcal{P}_{1243},
\end{aligned}
$$

and further using (4.23). The second and third relations follow similarly by considering $\mathcal{P}_{1243}$ (again!) and $\mathcal{P}_{2341}$ respectively. Thus, finally we are left with seven gauge-invariant polynomials of order two.

- Order 4 polynomials

Three cases arise for the polynomials $\mathcal{P}_{I J K L}$.

1. $\mathcal{P}_{I I J K}$ for all $I, J, K$. These are determined in terms of $\mathcal{P}_{I I}$ and $\mathcal{P}_{J K}$ by virtue of (5.6).

2. $\mathcal{P}_{I I J J}=\frac{1}{2} x_{I} x_{J}$

3. $\mathcal{P}_{1234}$ - this is an independent polynomial. Other order 4 polynomials with all indices different are determined in terms of $\mathcal{P}_{1234}$ and certain polynomials of order 2 .

As for the polynomials $\widetilde{\mathcal{P}}_{I J K L}$,

1. $\widetilde{\mathcal{P}}_{I I J K}$ for all $I, J$. These are determined in terms of $\widetilde{\mathcal{P}}_{I I}$ and $\widetilde{\mathcal{P}}_{J K}$ by virtue of $(5.6)$, and the latter are related to $\mathcal{P}_{I I}$ and $\mathcal{P}_{J K}$ in turn, again by (5.6).

2. $\widetilde{\mathcal{P}}_{I I J J}$ are also determined in terms of the are determined by $x_{I}$

$$
\begin{aligned}
\widetilde{\mathcal{P}}_{I I J J} & =\frac{1}{2} \widetilde{x}_{I} \widetilde{x}_{J} \\
& =\frac{1}{2} x_{I} x_{J} .
\end{aligned}
$$

3. $\widetilde{\mathcal{P}}_{1234}$ is determined in terms of $\mathcal{P}_{1234}$ by the relation

$$
\widetilde{\mathcal{P}}_{1234}=\mathcal{P}_{1234}+\frac{1}{2}\left(\xi_{1} \eta_{1}-\xi_{2} \eta_{2}+\xi_{3} \eta_{3}\right)
$$

as a consequence of the F-flatness conditions (4.23)

Thus, we conclude that, the only independent polynomial of order four is $\mathcal{P}_{1234}$. 
- Polynomials of order higher than 4

These can all be expressed in terms of polynomials of lower order. Some of these relations follow by (5.6) and (5.7), while the others follow by an use of the Cayley-Hamilton theorem. As an example of the latter cases, let us consider the polynomial $\mathcal{P}_{111134}=\frac{1}{2} \operatorname{Tr} z_{1} w_{1} z_{1} w_{1} z_{3} w_{4}$. The Cayley-Hamilton theorem leads to the following equation for any $2 \times 2$ matrix, $\mathbf{M}$.

$$
\mathbf{M}^{2}-\mathbf{M} \operatorname{Tr} \mathbf{M}+\frac{1}{2}\left((\operatorname{Tr} \mathbf{M})^{2}-\operatorname{Tr} \mathbf{M}^{2}\right) \mathbb{I}_{2}=0 .
$$

Now, taking $\mathbf{M}=z_{1} w_{1}$, we have

$$
z_{1} w_{1} z_{1} w_{1}-2 z_{1} w_{1} \mathcal{P}_{11}+\left(2 \mathcal{P}_{11}^{2}-\mathcal{P}_{1111}\right) \mathbb{I}_{2}=0
$$

Multiplying by $z_{3} w_{4}$ and taking trace we obtain

$$
\mathcal{P}_{112234}=2 \mathcal{P}_{11} \mathcal{P}_{1134}-2 \mathcal{P}_{11}^{2} \mathcal{P}_{34}+\mathcal{P}_{1111} \mathcal{P}_{34}
$$

Thus, the polynomial $\mathcal{P}_{112234}$ of order six is expressed in terms of polynomials of lower order. Other polynomials of order higher than four can be treated similarly. In particular, in any polynomial of order say $(4+k), k>0$, at least $k$ indices come repeated. Using (4.23) it is always possible to gather these repeated indices together and use the proportionality of $\mathcal{Q}_{I I}$ to the identity matrix to reduce it to some combination of lower order polynomials or use the Cayley-Hamilton theorem as above. Thus, finally we have ten independent, gauge invariant polynomials of order two and one of order four. The vacuum moduli space is described by an equation involving these polynomials upon using the F-flatness conditions.

\subsection{The variety}

Now, in order to write down the equation for the variety describing the moduli space, let us introduce a $4 \times 4$ matrix $\mathcal{M}$, defined as follows.

$$
\begin{aligned}
\mathcal{M}_{I I} & =\mathcal{P}_{I I}, \\
\mathcal{M}_{i j} & =\frac{1}{2}\left(\mathcal{P}_{i j}+\widetilde{\mathcal{P}}_{i j}\right), \\
\mathcal{M}_{i 4} & =\frac{1}{2}\left(\widetilde{\mathcal{P}}_{i 4}-\mathcal{P}_{i 4}\right), \\
\mathcal{M}_{4 i} & =\frac{1}{2}\left(\widetilde{\mathcal{P}}_{4 i}-\mathcal{P}_{4 i}\right) \\
& =-\mathcal{M}_{i 4},
\end{aligned}
$$

for $I, J=1,2,3,4$ ans $i, j=1,2,3$. Defining another complex variable $t$ as

$$
t=\frac{1}{2 i}\left(\mathcal{P}_{1234}+\widetilde{\mathcal{P}}_{1234}\right)
$$


the equation of the variety is written as

$$
t^{2}=\operatorname{det} \mathcal{M}
$$

We now write this in terms of $t$ and $x_{I}, I=1,2,3,4$ and use (4.23). The matrix $\mathcal{M}$ now assumes the following form:

$$
\mathcal{M}=\left(\begin{array}{cccc}
x_{1} & \xi_{3} / 2 & \xi_{2} / 2 & \eta_{1} / 2 \\
\xi_{3} / 2 & x_{2} & \xi_{1} / 2 & \eta_{2} / 2 \\
\xi_{2} / 2 & \xi_{1} / 2 & x_{3} & \eta_{3} / 2 \\
-\eta_{1} / 2 & -\eta_{2} / 2 & -\eta_{3} / 2 & x_{4}
\end{array}\right)
$$

The matrix $\mathcal{M}$ is the most general one consistent with the global $U(1)^{4}$ symmetry, provided we assign the correct charges to the parameters $\xi$ and $\eta$ from the action.

The relation (5.16) can be argued from the algebra of the matrices $\mathcal{Q}_{I J}$ that follows from (4.23). Any solution is a represenattion of the algebra and it can be shown from the algebra that in the generic case the moduli are related through $(5.16)$.

Hence the equation of the variety becomes

$$
\mathcal{F}\left(x_{1}, x_{2}, x_{3}, x_{4}, t\right)=0
$$

where we have defined

$$
\begin{aligned}
\mathcal{F}\left(x_{1}, x_{2}, x_{3}, x_{4}, t\right)=x_{1} x_{2} x_{3} x_{4} & -\frac{1}{16}\left(\xi_{1}^{2} \eta_{1}^{2}+\xi_{2}^{2} \eta_{2}^{2}+\xi_{3}^{3} \eta_{3}^{2}\right) \\
& +\frac{1}{8}\left(\xi_{1} \xi_{2} \eta_{1} \eta_{2}+\xi_{2} \xi_{3} \eta_{2} \eta_{3}+\xi_{3} \xi_{1} \eta_{3} \eta_{1}\right) \\
& -\frac{1}{4}\left(\xi_{1} \eta_{2} \eta_{3} x_{1}+\xi_{2} \eta_{3} \eta_{1} x_{2}+\xi_{3} \eta_{1} \eta_{2} x_{3}\right) \\
& +\frac{1}{4}\left(\eta_{3}^{2} x_{1} x_{2}+\eta_{1}^{2} x_{2} x_{3}+\eta_{2}^{2} x_{3} x_{1}\right) \\
& -\frac{1}{4}\left(\xi_{1}^{2} x_{1}+\xi_{2}^{2} x_{2}+\xi_{3}^{2} x_{3}-\xi_{1} \xi_{2} \xi_{3}\right) x_{4} \\
& -t^{2} .
\end{aligned}
$$

That the equation (5.18) is indeed satisfied by solutions of the F-flatness conditions should be explicitly checked on any solution of (4.23). Let us verify this in some examples.

\section{Example 1}

When $\xi_{i}=\eta_{i}=0$ for $i=1,2,3$, a solution of (4.23) is given by

$$
\begin{aligned}
z_{I} & =\mathrm{z}_{I} \sigma_{I} \\
w_{I} & =\mathrm{w}_{I} \sigma_{I}, \quad I=1,2,3,4,
\end{aligned}
$$


where $z_{I}$ and $w_{I}$ are complex numbers. The F-flatness constraints (4.23) impose the following relations among $\mathrm{z}_{I}$ and $\mathrm{w}_{I}$ :

$$
\begin{array}{ll}
\mathrm{z}_{1} \mathrm{w}_{2}=\mathrm{z}_{2} \mathrm{w}_{1}, & \mathrm{z}_{1} \mathrm{w}_{4}=\mathrm{z}_{4} \mathrm{w}_{1}, \\
\mathrm{z}_{2} \mathrm{w}_{3}=\mathrm{z}_{3} \mathrm{w}_{2}, & \mathrm{z}_{2} \mathrm{w}_{4}=\mathrm{z}_{4} \mathrm{w}_{2}, \\
\mathrm{z}_{3} \mathrm{w}_{1}=\mathrm{z}_{1} \mathrm{w}_{3}, & \mathrm{z}_{3} \mathrm{w}_{4}=\mathrm{z}_{4} \mathrm{w}_{3} .
\end{array}
$$

We also need the constraints ensuing from the D-flatness condition (4.10), namely

$$
\left|z_{1}\right|^{2}+\left|z_{2}\right|^{2}+\left|z_{3}\right|^{2}+\left|z_{4}\right|^{2}-\left|w_{1}\right|^{2}-\left|w_{2}\right|^{2}-\left|w_{3}\right|^{2}-\left|w_{4}\right|^{2}=0 .
$$

Let us note that only the three relations written in the second column of (5.21) are independent - the other three relations in the first column follow from these. Also, note that the three equations in the second column of (5.21) are monomial equations. Therefore, we can give a toric description to the envisaged variety, following [7]. In the notation of [10], we can express the variables $z_{I}, w_{I}$, in terms of the five independent variables, which we choose to be $z_{1}, z_{2}$, $z_{3}, z_{4}$ and $w_{4}$. This is expressed as:

$$
\begin{aligned}
& \begin{array}{llllllll}
\mathrm{z}_{1} & \mathrm{z}_{2} & \mathrm{z}_{3} & \mathrm{z}_{4} & \mathrm{w}_{1} & \mathrm{w}_{2} & \mathrm{w}_{3} & \mathrm{w}_{4}
\end{array} \\
& \begin{array}{l}
\mathrm{z}_{1} \\
\mathrm{z}_{2} \\
\mathrm{z}_{3} \\
\mathrm{z}_{4} \\
\mathrm{w}_{4}
\end{array}\left(\begin{array}{cccccccc}
1 & 0 & 0 & 0 & 1 & 0 & 0 & 0 \\
0 & 1 & 0 & 0 & 0 & 1 & 0 & 0 \\
0 & 0 & 1 & 0 & 0 & 0 & 1 & 0 \\
0 & 0 & 0 & 1 & -1 & -1 & -1 & 0 \\
0 & 0 & 0 & 0 & 1 & 1 & 1 & 1
\end{array}\right) .
\end{aligned}
$$

In terms of six homogeneous coordinates, $p_{i}, i=0, \cdots 5$, the five independent variables can then be expressed as:

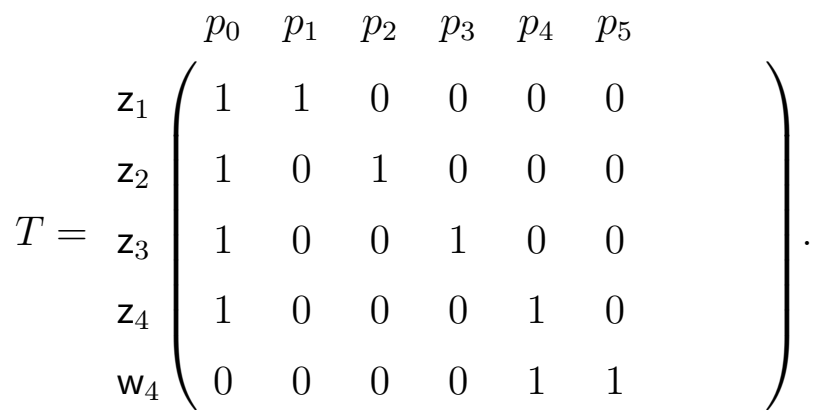

The kernel of $T$ is given by

$$
(\operatorname{Ker} T)^{\mathrm{T}}=\left(\begin{array}{llllll}
1 & -1 & -1 & -1 & -1 & 1
\end{array}\right),
$$


and provides part of the charge matrix for the variety. We can also find out the charges of the five independent variables under a $\mathbb{C}^{\star}$ from the D-flatness condition (5.22) as

$$
V=\left(\begin{array}{lllll}
1 & 1 & 1 & 1 & -1
\end{array}\right) .
$$

The charges of the homogeneous coordinates $p_{i}$ under this $\mathbb{C}^{\star}$ are obtained from $V$, using a matrix $U$, satisfying $T U^{\mathrm{T}}=\mathbb{I}_{5}$, namely,

$$
U=\left(\begin{array}{cccccc}
0 & 1 & 0 & 0 & 0 & 0 \\
0 & 0 & 1 & 0 & 0 & 0 \\
0 & 0 & 0 & 1 & 0 & 0 \\
1 & -1 & -1 & -1 & 0 & 0 \\
0 & 0 & 0 & 0 & 0 & 1
\end{array}\right)
$$

as follows:

$$
V U=\left(\begin{array}{llllll}
1 & 0 & 0 & 0 & 0 & -1
\end{array}\right) .
$$

Concatenating this with $(\operatorname{Ker} T)^{\mathrm{T}}$ in $(5.23)$, we obtain the charge matrix

$$
\widetilde{Q}=\left(\begin{array}{cccccc}
1 & -1 & -1 & -1 & -1 & 1 \\
1 & 0 & 0 & 0 & 0 & -1
\end{array}\right) .
$$

The co-kernel of the transpose of $\widetilde{Q}$, after deleting a column, identical to the first one, takes the form

$$
\widetilde{T}=\operatorname{coKer} \widetilde{Q}^{T}=\left(\begin{array}{lllll}
1 & 2 & 0 & 0 & 0 \\
1 & 0 & 2 & 0 & 0 \\
1 & 0 & 0 & 2 & 0 \\
1 & 0 & 0 & 0 & 2
\end{array}\right),
$$

and is the toric data of the variety under consideration. The equation of the variety can be read of from the kernel of $\widetilde{T}$, namely

$$
\text { Ker } \widetilde{T}=\left(\begin{array}{c}
2 \\
-1 \\
-1 \\
-1 \\
-1
\end{array}\right) \text {. }
$$

Correspondingly, the equation of the variety under consideration is given by

$$
t^{2}=x_{1} x_{2} x_{3} x_{4}
$$


which is the same as (5.18) with $\xi_{i}=\eta_{i}=0$. The five variables appearing in the above equation are expressed in terms of the gauge-invariant combinations described earlier, as follows.

$$
\begin{aligned}
x_{I} & =\mathrm{z}_{I} \mathrm{w}_{I}, \quad I=1,2,3,4, \\
t & =\frac{1}{2}\left(\mathrm{z}_{1} \mathrm{w}_{2} \mathrm{z}_{3} \mathrm{w}_{4}+\mathrm{w}_{1} \mathrm{z}_{2} \mathrm{w}_{3} \mathrm{z}_{4}\right) .
\end{aligned}
$$

Thus, we have verified the claim that $(5.18)$ is indeed the equation of the variety describing the vacuum moduli space.

Let us now verify the claim in the case where all the deformation parameters are non-vanishing. We wish to have a solution which goes over to the solution given in the previous example in the limit of vanishing deformation parameters.

\section{Example 2}

Let us present the solution first.

$$
\begin{aligned}
z_{1}=\mathrm{z}_{1} \sigma_{1}+\xi_{3} \sigma_{2} / 2 \mathrm{w}_{2}, & w_{1}=\mathrm{w}_{1} \sigma_{1}+\xi_{3} \sigma_{2} / 2 \mathrm{z}_{2}, \\
z_{2}=\mathrm{z}_{2} \sigma_{2}+\xi_{1} \sigma_{3} / 2 \mathrm{w}_{3}, & w_{2}=\mathrm{w}_{2} \sigma_{2}+\xi_{1} \sigma_{3} / 2 \mathrm{z}_{3}, \\
z_{3}=\mathrm{z}_{3} \sigma_{3}+\xi_{2} \sigma_{1} / 2 \mathrm{w}_{1}, & w_{3}=\mathrm{w}_{3} \sigma_{3}+\xi_{2} \sigma_{1} / 2 \mathrm{z}_{1}, \\
z_{4}=\mathrm{z}_{4} \sigma_{4}+\alpha_{1} \sigma_{1}+\alpha_{2} \sigma_{2}+\alpha_{3} \sigma_{3}, & w_{4}=\mathrm{w}_{4} \sigma_{4}+\ell\left(\alpha_{1} \sigma_{1}+\alpha_{2} \sigma_{2}+\alpha_{3} \sigma_{3}\right),
\end{aligned}
$$

where $\mathrm{z}_{I}$ and $\mathrm{w}_{I}$ satisfy the equations (5.21) and the three complex variables $\alpha_{1}, \alpha_{2}, \alpha_{3}$ solve the following set of three relations:

$$
\begin{aligned}
& 2 \mathrm{z}_{1} \mathbf{w}_{3} \alpha_{3}+\xi_{2} \alpha_{1}-\eta_{3} \mathbf{z}_{1}=0 \\
& 2 \mathbf{z}_{2} \mathbf{w}_{1} \alpha_{1}+\xi_{3} \alpha_{2}-\eta_{1} \mathbf{z}_{2}=0 \\
& 2 \mathrm{z}_{3} \mathbf{w}_{2} \alpha_{2}+\xi_{1} \alpha_{3}-\eta_{2} \mathbf{z}_{3}=0
\end{aligned}
$$

and we have defined

$$
\ell=-\frac{\mathrm{w}_{1}}{\mathrm{z}_{1}}=-\frac{\mathrm{w}_{2}}{\mathrm{z}_{2}}=-\frac{\mathrm{w}_{3}}{\mathrm{z}_{3}}=-\frac{\mathrm{w}_{4}}{\mathrm{z}_{4}}
$$

by (5.21). It should be noted that $z_{I}$ and $w_{I}$ as given in (5.32), satisfy the F-flatness conditions (4.23) if $z_{I}$ and $w_{I}$ satisfy (5.21). Hence, as above, the corresponding variety is again a fourfold, given by (5.18) in $\mathbb{C}^{5}$. However, the $D$-flatness conditions alter from (4.10). In terms of the 
above solution, we have

$$
\begin{aligned}
x_{1} & =\mathrm{z}_{1} \mathrm{w}_{1}+\frac{\xi_{3}^{2}}{4 \mathrm{z}_{2} \mathrm{w}_{2}}, \\
x_{2} & =\mathrm{z}_{2} \mathrm{w}_{2}+\frac{\xi_{1}^{2}}{4 \mathrm{z}_{3} \mathrm{w}_{3}}, \\
x_{3} & =\mathrm{z}_{3} \mathrm{w}_{3}+\frac{\xi_{2}^{2}}{4 \mathrm{z}_{1} \mathrm{w}_{1}}, \\
x_{4} & =\mathrm{z}_{4} \mathrm{w}_{4}+\ell\left(\alpha_{1}^{2}+\alpha_{2}^{2}+\alpha_{3}^{2}\right), \\
t & =\left(\mathrm{z}_{1} \mathrm{w}_{2} \mathrm{z}_{3} \mathrm{w}_{4}+\frac{\xi_{1} \xi_{2} \xi_{3} \mathrm{w}_{4}}{8 \mathrm{w}_{1} \mathrm{z}_{2} \mathrm{w}_{3}}\right) .
\end{aligned}
$$

We have used (5.21) in writing the expression for $t$ in (5.35). The equation (5.18) is satisfied for these values of the five variables.

Let us note that, as mentioned above, we have the same equations among the different variables $\mathrm{z}_{I}$ and $\mathrm{w}_{I}$ in the present case, as in Example 1 above. However, the D-flatness conditions are now different and involves the parameters $\xi_{i}$ and $\eta_{i}$. We have not solved the D-flatness equations. However, the above solution should be gauge-equivalent to a solution that simultaneously solves the F- and D-flatness equations [22]. The variety, anyway, is different from the one in the previous example.

\subsection{Special branches}

Returning to the equation (5.18) for the variety, it has a variety of singularities depending on the values of the deformation parameters $\xi_{i}$ and $\eta_{i}$. The singular subsets of the variety (5.18) are simultaneous solutions of $\mathcal{F}=0$ and $\partial \mathcal{F}=0$, as noted in $\S$. We list some of the cases below.

NB: In this subsection we have rescaled the parameters $\xi_{i}$ and $\eta_{i}$ to $2 \xi_{i}$ and $2 \eta_{i}$ respectively, in order to avoid clumsy factors in the expressions.

1. In the limit of vanishing deformation, $\xi_{i}=\eta_{i}=0$, we recover the singular orbifold. This has a $\mathbb{Z}_{2} \times \mathbb{Z}_{2} \times \mathbb{Z}_{2}$ singularity at the origin, along with higher dimensional singular subspaces, all of which contain the origin.

2. When all $\xi_{i}=0$, we can have one, two or all three of the $\eta_{i}$ non-vanishing.

(a) $\eta_{i} \neq 0$. This has a line singularity along $x_{4}$, with $x_{1}=x_{2}=x_{3}=t=0$.

(b) $\eta_{1}=0, \eta_{2}, \eta_{3} \neq 0$. This also has a line singularity along $x_{4}$, with $x_{i}=t=0$.

(c) $\eta_{1}, \eta_{2} \neq 0, \eta_{3}=0$. This has a singular plane given by $x_{3}=0, x_{1} x_{2} x_{4}+\eta_{1}^{2} x_{2}+\eta_{2}^{2} x_{1}=0$.

(d) $\eta_{1}=\eta_{2}=0, \eta_{3} \neq 0$. The singularity is along the union of the two planes given by: $x_{3}-x_{4}$ plane, with $x_{1}=x_{2}=t=0$, and the plane, $x_{1}=0, x_{3} x_{4}+\eta_{3}^{2}=0, x_{2}$ arbitrary. 
3. The next cases are when one of the $\xi_{i}$ is non zero. Let us assume, $\xi_{1}=\xi_{2}=0, \xi_{3} \neq 0$. we can have different numbers and combinations of $\eta_{i}$ non-vanishing.

(a) $\eta_{i} \neq 0$. This has a line singularity given by

$$
x_{1}=\frac{\xi_{3} \eta_{1}}{\eta_{2}}, \quad x_{2}=\frac{\xi_{3} \eta_{2}}{\eta_{1}}, \quad x_{3} x_{4}+\frac{\eta_{1} \eta_{2}}{\xi_{3}} x_{3}+\eta_{3}^{2}=0
$$

(b) $\eta_{1}, \eta_{2} \neq 0, \eta_{3}=0$. This has a singular line which can be obtained by setting $\eta_{3}=0$ in (5.36). The case with $\xi_{i} \neq 0, \eta_{i}=0$ will be similar.

(c) $\eta_{1}=0$ and $\eta_{2}, \eta_{3} \neq 0$. There is no singularity. This can also be seen by taking the limit $\eta_{1} \longrightarrow 0$ in $(5.36),-$ the singularity is sent to infinity along $x_{2}$.

(d) The case with $\eta_{2}=0, \eta_{1}, \eta_{3} \neq 0$ is similar to (3c).

(e) $\eta_{1} \neq 0, \eta_{2}=\eta_{3}=0$. This has a line singularity along $x_{1}$ with $x_{2}=x_{3}=x_{4}=0$. This is similar to the case with $\eta_{2} \neq 0, \eta_{3} \neq 0$ and the rest of the parameters vanishing.

(f) $\eta_{1}=\eta_{2}=0, \eta_{3} \neq 0$. This has singularity along a plane given by the common solution of

$$
x_{1} x_{2}-\xi_{3}^{2}=0, \quad x_{3} x_{4}+\eta_{3}^{2}=0
$$

4. Next, let us consider the cases, when two of the $\xi_{i}$ are non-vanishing, say $\xi_{1}, \xi_{2} \neq 0, \xi_{3}=0$.

(a) When all $\eta_{i} \neq 0$, the variety has a singular line given by

$$
x_{1} x_{2}=0, \quad x_{3}=\xi_{1}^{2} x_{1}+\xi_{2}^{2} x_{2}, \quad x_{4}=-\left(\eta_{1}^{2} x_{2}+\eta_{2}^{2} x_{1}\right)
$$

The case with $\xi_{i} \neq 0, \eta_{1}=0, \eta_{2}=0$ will be similar.

(b) $\eta_{1}, \eta_{2} \neq 0, \eta_{3}=0$. There is no singularity.

(c) $\eta_{1}=0, \eta_{2}, \eta_{3} \neq 0$. This has a line-singularity given by

$$
\begin{aligned}
x_{2}=\frac{\eta_{2} \xi_{1}}{\eta_{3}}, \quad x_{4} & =-\frac{\eta_{2} \eta_{3}}{\xi_{1}} \\
\eta_{2}\left(x_{1} x_{3}-\xi_{2}^{2}\right)-\eta_{3} \xi_{1} x_{1} & =0
\end{aligned}
$$

(d) $\eta_{1}=\eta_{2}=0, \eta_{3} \neq 0$. This has a singularity along $x_{3}$, with $x_{1}=x_{2}=x_{4}=0$.

(e) $\eta_{1} \neq 0, \eta_{2}, \eta_{3}=0$. This has a singular plane along the solutions of

$$
x_{1} x_{4}+\eta_{1}^{2}=0, \quad x_{2} x_{3}-\xi_{1}^{2}=0
$$


5. Finally, the cases with all the three $\xi_{i}$ turned on. When all $\eta_{i} \neq 0$, this has a line singularity

$$
\begin{aligned}
& x_{1}=\frac{\xi_{2} \xi_{3}}{\xi_{1}}\left[1+\frac{(a-b)(c-a)}{x+b c}\right], \\
& x_{2}=\frac{\xi_{3} \xi_{1}}{\xi_{2}}\left[1+\frac{(a-b)(b-c)}{x+c a}\right], \\
& x_{3}=\frac{\xi_{1} \xi_{2}}{\xi_{3}}\left[1+\frac{(b-c)(c-a)}{x+a b}\right], \\
& x_{4}=\frac{x}{\xi_{1} \xi_{2} \xi_{3}} \\
& t=0
\end{aligned}
$$

where we have defined $a=\eta_{1} \xi_{1}, b=\eta_{2} \xi_{2}$ and $c=\eta_{3} \xi_{3}$ and $x$ is an arbitrary complex number.

The different cases with some of the $\eta_{i}$ set to zero can be obtained from (5.41) by setting the parameters $a, b, c$ to zero accordingly.

Moreover, in the above discussion, we have assumed generic values of the six parameters $\xi_{i}$ and $\eta_{i}$, whenever non-zero. Several other special branches can be obtained by relating these non-vanishing parameters. These can be obtained from the corresponding cases in the above list.

\subsection{The moduli space for other representations of $G$}

So far we have used the eight-dimensional regular representation of $G$, constructed by tensoring the four-dimensional projective representation $\operatorname{Diag}\left\{\sigma_{I},-\sigma_{I}\right\}$, from (2.6) with $\mathbb{I}_{M}$, with $M=2$. This corresponds to a single brane at the orbifold singularity. The theory with $N$ branes at the singularity can be obtained, in a similar way as above, using $\mathbb{I}_{M}$, such that $4 M=2^{3} N$. The moduli spaces for configurations with $N>1$ branes, or equivalently $M>2$, can be found in the same manner as discussed above. However, it has been pointed out [4,5] that string theory allows also the case with $M=1$. Let us discuss this case briefly in this subsection.

The quantities $z_{I}$ and $w_{I}$ appearing in (4.14) are now numbers, instead of $2 \times 2$ matrices. The $M=1$ moduli space ensues by imposing the F-flatness conditions (4.23) on the complex numbers $z_{I}$ and $w_{I}$.

We can define the gauge-invariant polynomials as above. However, in the present case, the polynomials $z_{1} w_{2} z_{3} w_{4}$ and $w_{1} z_{2} w_{3} z_{4}$ are not independent, but determined by polynomials of order two, e.g. $2 z_{1} w_{2} z_{3} w_{4}=\frac{1}{2}(a-b+3 c)+\xi_{3} z_{3} w_{4}+\eta_{3} z_{1} w_{2}$. Thus, we define only the four variables

$$
x_{1}=z_{I} w_{I}, \quad I=1,2,3,4
$$

as above. 
When $\eta_{i}=\xi_{i}=0$, we have, the F-flatness equations (4.23), can be partially solved with, e.g. $z_{1}=$ $z_{2}=w_{1}=w_{2}=0$. This leaves us with the following F-flatness condition

$$
z_{3} w_{4}-z_{4} w_{3}=0
$$

and the D-flateness equation, now reduced to

$$
\left|z_{3}\right|^{2}+\left|z_{4}\right|^{2}-\left|w_{3}\right|^{2}-\left|w_{4}\right|^{2}=0
$$

This space admits a toric description, thanks to the monomial relation (5.43). Following the steps outlined in Example 1 above, it can be seen that the moduli space is a $\mathbb{C}^{2} / \mathbb{Z}_{2}$-plane

$$
x z-y^{2}=0
$$

where $x, y, z$ are three complex numbers. The same exercise can be repeated with other pairs of $z_{i}$ and $w_{i}$, corresponding to other possible solutions of the F-flatness equations. Since there are six such solutions, six $\mathbb{C}^{2} / \mathbb{Z}_{2}$-planes arise and the moduli space is given as the union of these six planes. These six planes can be interpreted as associated with the invariant subspaces of the six group elements each of which contribute a deformation. The fractional brane may be a D3-brane wrapped on a vanishing two-sphere transverse to the plane. However a confirmmation of this needs more involved analysis.

When $\eta_{i}=0$, and $\xi_{i} \neq 0$, three of the equations in (4.23) involving $z_{4}$ and $w_{4}$ are solved by $w_{I}=s z_{I}$, where $s$ is a constant. The three rest can be rewritten as

$$
\begin{aligned}
& x_{1} x_{2}=\xi_{3}^{2} / 4, \\
& x_{2} x_{3}=\xi_{1}^{2} / 4, \\
& x_{3} x_{1}=\xi_{2}^{2} / 4,
\end{aligned}
$$

while $x_{4}=s z_{4}^{2}$. The solution to these equations furnishes the moduli space, which is a line given by

$$
x_{1}=\frac{\xi_{2} \xi_{3}}{2 \xi_{1}}, \quad x_{2}=\frac{\xi_{3} \xi_{1}}{2 \xi_{2}}, \quad x_{3}=\frac{\xi_{1} \xi_{2}}{2 \xi_{3}},
$$

with $x_{4}$ arbitrary.

Finally, when all $\xi_{i}$ and $\eta_{i}$ are non-zero, the moduli space is described by equations involving $x_{i}$, $i=1,2,3$ in a similar, but more complicated way, as above. These can again be solved for $x_{i}$, while $x_{4}$ is left arbitrary. Thus, the moduli space is again a line.

\section{Conclusion}

To summarise, we have studied a D1-brane on the four-dimensional orbifold singularity $\mathbb{C}^{4} /\left(\mathbb{Z}_{2} \times \mathbb{Z}_{2} \times\right.$ $\mathbb{Z}_{2}$ ), with discrete torsion. The resulting moduli space in absence of any deformation is the singular 
orbifold given by the equation $t^{2}=x_{1} x_{2} x_{3} x_{4}$ in $\mathbb{C}^{5}$, as found earlier [13]. The deformations of the moduli space in the presence of discrete torsion correspond to perturbations of the superpotential of the corresponding $(0,2)$ SYM in two dimensions, and are constrained by consistency requirements from string theory on the orbifold. The moduli arising in the twisted sector of string theory now deform away certain singularities of codimensions one and two, which in turn correspond to some subsets of $\mathbb{C}^{4}$ fixed by certain elements of the group $G=\mathbb{Z}_{2} \times \mathbb{Z}_{2} \times \mathbb{Z}_{2}$. This is in harmony with expectations from conformal field theoretic description. But the singularity with codimension three turns out to be stable, as the twisted sector of the closed string theory fails to provide the modes required for its deformation. That the stable singularity is a line and not a point, unlike its three-

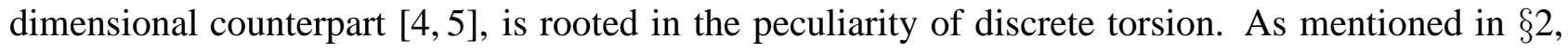
the discrete torsion $\alpha$, in spite of its deceptive general appearance in (2.5), is actually between two $\mathbb{Z}_{2}$ factors out of the three in the quotienting group $\mathbb{Z}_{2} \times \mathbb{Z}_{2} \times \mathbb{Z}_{2}$. By a suitable change of basis, we can thus think of the discrete torsion as affecting only a $\mathbb{Z}_{2} \times \mathbb{Z}_{2}$ subgroup of $G$, acting on a subset $\mathbb{C}^{3} \subset \mathbb{C}^{4}$. Apart from the details of further quotienting by a $\mathbb{Z}_{2}$, it is the node of this $\mathbb{C}^{3} /\left(\mathbb{Z}_{2} \times \mathbb{Z}_{2}\right)$ [由, Б], that gives rise to the singular line.

Indeed, this aspect of discrete torsion has featured in studies of mirror symmetry on this orbifold. Starting from the Calabi-Yau manifold obtained as the blown up $\mathbb{T}^{8} /\left(\mathbb{Z}_{2} \times \mathbb{Z}_{2} \times \mathbb{Z}_{2}\right)$, which is the compact version of our case at hand, one can T-dualise the type-II string theory on this manifold along the $\mathbb{T}^{4}$-fibres, to obtain the same string theory on the deformed $\mathbb{T}^{8} /\left(\mathbb{Z}_{2} \times \mathbb{Z}_{2} \times \mathbb{Z}_{2}\right)$ singularity. The T-dualities along the four directions of the $\mathbb{T}^{4}$ administers the "right dose" of discrete torsion as in here, such that the two theories are mirror-dual to each other. This situation provides a nontrivial demonstration of mirror symmetry [16]. Thus, the situation considered in the present article is expected to be mirror-dual to the moduli space obtained by considering D-brane in absence of discrete torsion [13]. A comment is in order. In absence of discrete torsion, a D1-brane on the orbifold $\mathbb{C}^{4} /\left(\mathbb{Z}_{2} \times \mathbb{Z}_{2} \times \mathbb{Z}_{2}\right)$ resolves the orbifold singularity with seven Fayet-Iliopoulos parameters [13]. In view of the fact that for this resolution, $h^{11}=6$, as we found in $\S$, this signifies that the resolved moduli space is smooth but not Calabi-Yau [19]. It is not clear at the moment if one should invoke the mirror principle beyond Calabi-Yau varieties [23] to incorporate another parameter of deformation in the present case also, superceding the restrictions arising from the twisted sector of string theory. If so, then this will call for a formulation of consistency conditions beyond the stringy ones studied here.

Another implication of this configuration is related to the fact that discrete torsion can be simulated through an antisymmetric B-field background [1]. On $\mathbb{C}^{3} /\left(\mathbb{Z}_{2} \times \mathbb{Z}_{2}\right)$, the B-field has a non-zero field strength supported at the singular point as necessitated by supersymmetry [2]. In the present case, by an S-duality transformation, we may change the D-string into a fundamental string (in Type-IIB) and the background NS-NS B-field to an RR B-field. Thus, the present analysis can also be interpreted as describing a fundamental string at an orbifold in presence of a background RR B field which has a 
non-zero field strength only on a line singularity at the classical level.

Finally, in the case of orbifold singularities there exist parallel brane configurations that give rise to the theory of branes at the singularity. In particular, one can also map the desingularisation moduli of the singularity to the parameters of the brane configurations [24, 25]. It would be interesting to understand the analogous brane configurations corresponding to present case and identify the presence as well as the absence of the various deformation modes, which will provide another way of looking at the orbifolds with discrete torsion.

\section{Acknowledgement}

We would like to thank D Jatkar and A Sen for illuminating discussions.

\section{References}

[1] C Vafa. Modular invariance and discrete torsion on orbifolds; Nucl. Phys. B273 (1986) 592.

[2] C Vafa and E Witten. On orbifolds with discrete torsion; J. Geom. Phys. 15 (1995) 189; hepth/9409188.

[3] P Aspinwall. Resolution of Orbifold Singularities; hep-th/9403123.

[4] M Douglas. D-branes and discrete torsion-I; hep-th/9807235.

[5] M Douglas and B Fiol. D-branes and discrete torsion-II; hep-th/9903031.

[6] M Douglas and G Moore. D-branes, Quivers and ALE instantons; hep-th/9603167.

[7] M Douglas, B Greene and D Morrison. Orbifold resolution by D-branes; Nucl. Phys. B506 (1997) 84; hep-th/9608024.

[8] C Johnson and R Myers. Aspects of Type IIB theory on ALE spaces; Phys. Rev. D55 (1997) 6382: hep-th/9610140.

[9] B Greene. D-brane topology changing transition; Nucl. Phys. B525 (1998) 284; hepth/9711124.

[10] S Mukhopadhyay and K Ray. Conifolds from D-brane; Phys. Lett. B423 (1998) 247; hepth/9711131.

[11] D Morrison and M Plesser. Non-spherical horizons I; Adv. Theor. Math. Phys. 3 (1999) 1; hepth/9810201. 
[12] D Diaconescu and J Gomis. Fractional branes and boundary states in orbifold theories; hepth/9906242.

[13] $\mathrm{C}$ Ahn and $\mathrm{H}$ Kim. Branes from $\mathbb{C}^{4} / \Gamma$ singularity from toric geometry; JHEP 9904 (1999) 012; hep-th/9903181.

[14] P Aspinwall and D Morrison. Stable singularities in string theory; Comm. Math. Phys.178 (1996) 115; hep-th/9503208.

[15] G Karpilovsky. Projective Representations of finite Groups; Marcel Dekker, Inc. 1985.

[16] B Acharya. A Mirror pair of Calai-Yau Four-folds in type II String Theory; Nucl. Phys. B524 (1998) 283; hep-th/9703029.

[17] E Zaslow. Topological orbifold models and quantum cohomology ring; Comm. Math. Phys.156 (1993) 301.

[18] K Mohri. Kähler moduli space for a D-brane at orbifold singularities; Comm. Math. Phys.202 (199) 669; hep-th/9806052.

[19] K Mohri. D-branes and quotient singularities of Calabi-Yau fourfolds; Nucl. Phys. B521 (1998) 161; hep-th/9707012.

[20] E Witten. Phases of N=2 theories in two dimensions; Nucl. Phys. B403 (1993) 159; hepth/9301042.

[21] J Distler. Notes on $(0,2)$ superconformal field theories; hep-th/9502012.

[22] M Luty and W Taylor. Varieties af vacua in classical supersymmetric gauge theories; Phys. Rev. D53 (1996) 3399; hep-th/9506098.

[23] V Batyrev. Stringy Hodge numbers of varieties with Gorenstein canonical singularities; alggeom/9711008.

[24] M Aganagic, A Karch, D Lüst, A Miemiec. Mirror symmetries for brane configurations and branes at singularities; hep-th/9903093.

[25] K Oh and R Tatar. Branes at orbifolded conifold singularities and supersymmetric gauge field theories; hep-th/9906012. 